\title{
A hemoglobina glicada e o laboratório clínico
}

A associação entre a hemoglobina glicada (A1C) e o diabetes mellitus (DM) já é conhecida desde a década de $1960^{(9)}$. No entanto, a real utilidade deste parâmetro laboratorial passou a ser reconhecida após a publicação de dois importantes estudos: Diabetes Control and Complications Trial (DCCT), de 1993(2), e United Kingdom Prospective Diabetes Study (UKPDS), de 1998(12). Desde então, os métodos para a dosagem da A1C evoluíram e o custo da determinação sofreu queda significativa em razão do uso crescente deste exame no controle do diabetes.

Em 1996 foi criado o National Glycohemoglobin Standardization Program (NGSP), patrocinado, em parte, pela American Diabetes Association (ADA) com a finalidade de comparar e certificar os diversos métodos utilizados para dosagem da $\mathrm{A} 1 \mathrm{C}^{(8)}$.

O método, uma vez certificado pelo NGSP, apresenta desempenho análogo e resultado semelhante aos do método aplicado no estudo do DCCT. Nessa condição, o resultado pode ser comparado ao valor considerado adequado para o controle do diabetes, estabelecido por estes estudos, que é de até $7 \%(1,2,8,12)$ ou, mais recentemente, de até $6,5 \%$, conforme a Sociedade Brasileira de Diabetes (SBD) $)^{(5)}$. Anualmente, os conjuntos diagnósticos devem passar pelo processo de certificação ${ }^{(8)}$. A lista atualizada mensalmente pode ser consultada no site www.ngsp.org.

É desejável que os laboratórios utilizem métodos certificados pelo NGSP(3-5, 8), e é importante ressaltar que a cromotografia líquida de alta eficiência (HPLC) não é o único método disponível para dosagem da hemoglobina glicada. Vários outros conjuntos diagnósticos que utilizam métodos não-HPLC também são certificados. Os seguintes métodos também foram avaliados pelo NGSP: imunoensaio turbidimétrico, eletroforese, cromatografia de troca iônica e enzimático(8).

A participação do laboratório em programas de ensaio de proficiência é fundamental para garantir a qualidade do resultado ${ }^{(3-5)}$. No Brasil, o Programa de Excelência para Laboratórios Médicos (PELM) da Sociedade Brasileira de Patologia Clínica/Medicina Laboratorial (SBPC/ML) oferece um programa específico para avaliação de desempenho em hemoglobina glicada.

As metodologias atualmente disponíveis para a dosagem da A1C apresentam elevados níveis de exatidão e reprodutibilidade ${ }^{(1,10,11)}$. No entanto, fatores interferentes podem resultar em valores não-compatíveis com o real estado de controle ou descontrole do diabetes $(1,3-5,7,10,11)$. Podemos citar como exemplo a limitação da dosagem da A1C nos pacientes portadores de hemoglobinas variantes (hemoglobinas C, S, E, D etc.), seja na forma heterozigótica ou homozigótica. Na homozigótica não é possível avaliar a A1C em razão da ausência da hemoglobina A. Na forma heterozigótica, alguns métodos baseados na cromatografia por troca iônica podem identificar a presença de alguns tipos de hemoglobina variante, permitindo uma análise mais criteriosa do resultado. É importante que o laboratório, ao selecionar o método de ensaio, considere o risco potencial das interferências e a prevalência das moléstias no grupo populacional avaliado $(1,3-5,7,10,11)$.

Finalmente, a incorporação gradativa do conceito da glicose média estimada, junto aos médicos e diabéticos, está sendo estimulada com a finalidade de complementar a informação clínica obtida mediante o resultado de hemoglobina glicada. A transformação do valor da A1C, em termos de glicose média, facilita sobremaneira sua interpretação em relação ao controle do diabetes e ao risco de desenvolvimento das complicações crônicas ${ }^{(5,6)}$. Esse valor é obtido por meio de uma equação matemática estabelecida pelo grupo de estudo denominado A1CDerived Average Glucose (ADAG) ${ }^{(6)}$, onde: glicose média estimada $(\mathrm{mg} / \mathrm{dl})=28,7 \times \mathrm{A} 1 \mathrm{C}-46,7$.

As entidades que participaram da elaboração do $3 \circ$ Posicionamento Oficial esperam contribuir para a melhoria da qualidade de vida dos diabéticos e estimular o uso e o entendimento desta importante ferramenta para o controle do diabetes. O grupo preocupou-se, na medida do possível, em utilizar linguagem simples com o objetivo de oferecer ao leitor um texto conciso e de fácil compreensão. 


\section{Referências}

1. AMERICAN DIABETES ASSOCIATION POSITION STATEMENT. Tests of glycemia in diabetes. Diabetes Care, v.27, supl. 1 p. S91-S3, 2004.

2. DCCT RESEARCH GROUP. Diabetes Control and Complications Trial (DCCT). The effect of intensive treatment of intensive treatment of diabetes on the development and progression of long-term complications in insulindependent diabetes mellitus. N Engl J Med, v. 329, p. 977-86, 1993.

3. GRUPO INTERDISCIPLINAR DE PADRONIZAÇÃO DA HEMOGLOBINA GLICADA (A1C). Hemoglobina glicada. Posicionamento Oficial (versão 2003). A importância da hemoglobina glicada (A1C) para a avaliação do controle glicêmico em pacientes com diabetes mellitus: aspectos clínicos e laboratoriais. Disponível em: http://www.sbpc. org. br/profissional/noticia.diverso. php?id=8\&tp=3. Acesso em: 26 abr 2009.

4. GRUPO INTERDISCIPLINAR DE PADRONIZAÇÃO DA HEMOGLOBINA GLICADA (A1C). Hemoglobina glicada. A importância da hemoglobina glicada (A1C) para a avaliação do controle glicêmico em pacientes com diabetes mellitus: aspectos clínicos e laboratoriais. Disponível em: http://www.sbpc.org.br/profissional/noticia.diverso. php?id=5\&tp=3. Acesso em: 26 abr 2009.

5. GRUPO INTERDISCIPLINAR DE PADRONIZAÇÃO DA HEMOGLOBINA GLICADA (A1C). Posicionamento Oficial (versão 2009). Atualização sobre hemoglobina glicada (A1C) para avaliação do controle glicêmico e para o diagnóstico do diabetes: aspectos clínicos e laboratoriais. Disponível em: <http://www.sbpc.org.br/profissional/ noticia. diverso. php?id=5\&tp=3>. Acesso em: 26 abr 2009.

6. NATHAN, D. M. et al. Translationg the A1C assay into estimated average glucose values. Diabetes Care, v. 31, p. 1473-8, 2008.

7. NATIONAL GLYCOHEMOGLOBIN STANDARDIZATION PROGRAM (NGSP). Factors that interfere with GHB (HbA1C) test results. Updated 4/08. Disponível em: <http://www.ngsp.org/prog/factors.htm>. Acesso em: 26 abr 2009.

8. NATIONAL GLYCOHEMOGLOBIN STANDARDIZATION PROGRAM (NGSP). IFCC standardization of HbA1C. Disponível em: <http://www.ngsp.org/prog/IFCCmain.html>. Acesso em: 26 abr 2009.

9. RAHBAR, S.; BLUMENFELD, O.; RANNEY, H. M. Studies of an unusual hemoglobin in patients with diabetes mellitus. Biochem Biophys Res Commun, v. 36, p. 838-43, 1969.

10. SUMITA, N. M.; ANDRIOLO, A. Importância da determinação da hemoglobina glicada no monitoramento do paciente portador de diabetes mellitus. J Bras Patol Med Lab, v. 42, editorial, 2006.

11. SUMITA, N. M.; ANDRIOLO, A. Importância da hemoglobina glicada no controle do diabetes mellitus e na avaliação de risco das complicações crônicas. J Bras Patol Med Lab, v. 44, p. 169-74, 2008.

12. UK PROSPECTIVE DIABETES STUDY GROUP. Intensive blood glucose control with sulphonylureas or insulin compared with conventional treatment and risk of complications in patients with type 2 diabetes. Lancet, $v$. 352, p. 837-53, 1998.

\section{Nairo Massakazu Sumita}

Médico patologista clínico; professor assistente doutor da disciplina de Patologia Clínica da Faculdade de Medicina da Universidade de São Paulo (FMUSP); diretor do Serviço de Bioquímica Clínica da Divisão de Laboratório Central do Hospital das Clínicas da FMUSP (LIM-03 da

Patologia Clínica); assessor médico em Bioquímica Clínica do Fleury Medicina e Saúde; membro do Grupo Interdisciplinar de Padronização da Hemoglobina Glicada - A1C. 